\title{
Endothelial dysfunction is associated with the severity of cerebral small vessel disease
}

\author{
Tomohisa Nezu ${ }^{1}$, Naohisa Hosomi ${ }^{1}$, Shiro Aoki ${ }^{1}$, Satoshi Kubo ${ }^{1}$, Mutsuko Araki ${ }^{1}$, Tomoya Mukai ${ }^{1}$, \\ Tetsuya Takahashi ${ }^{1}$, Hirofumi Maruyama ${ }^{1}$, Yukihito Higashi ${ }^{2}$ and Masayasu Matsumoto ${ }^{1}$
}

The pathogenesis of cerebral small vessel disease, a disease that involves white matter lesions (WMLs) and cerebral microbleeds (CMBs), is thought to be associated with endothelial dysfunction. Flow-mediated dilation (FMD) has been used to measure endothelium-dependent vasodilation. The aim of this study was to investigate the association between endothelial function (as measured by FMD) and cerebral small vessel disease. Patients with a history of cerebrovascular disease and comorbidities were enrolled in this study ( $n=102 ; 69$ males, $70.1 \pm 9.2$ years). The patients were divided into two groups according to the severity of WMLs, which were assessed by Fazekas classification; grades 0 to 1 as mild WMLs group and grades 2 to 3 as severe WMLs group. A gradient-echo MRI was performed in 96 patients (94.1\%) to evaluate whether CMBs were present. The patients in the severe WMLs group $(n=40)$ were older $(P=0.001)$, more frequently exhibited hypertension $(P=0.045)$ and diabetes mellitus $(P=0.026)$ and possessed lower FMD values $(P<0.001)$ than the patients in the mild WMLs group $(n=62)$. CMBs were observed in 30 patients $(31.3 \%)$. Using receiver operating characteristic curves, the optimal FMD cutoff values for predicting the presence of severe WMLs and CMBs were $3.9 \%$ and $3.7 \%$, respectively. On multivariate logistic analysis, FMD $<4.0 \%$ (odds ratio 9.50; $95 \%$ confidence interval 3.55-28.83) was independently associated with severe WMLs. Additionally, FMD $<3.8 \%$ (5.82; 2.23-16.50) was also associated with the presence of CMBs. Endothelial dysfunction as evaluated by FMD may be predictive of the severity of cerebral small vessel disease.

Hypertension Research (2015) 38, 291-297; doi:10.1038/hr.2015.4; published online 12 February 2015

Keywords: cerebral microbleeds; cerebral small vessel disease; endothelial dysfunction; white matter lesions

\section{INTRODUCTION}

Cerebral small vessel diseases such as white matter lesions (WMLs), lacunar infarcts and cerebral microbleeds (CMBs) are known to cause cognitive impairment, dementia and disability. ${ }^{1-3}$ Additionally, patients with cerebral small vessel diseases have an elevated long-term risk of stroke recurrence and unfavorable stroke outcomes. $^{4,5}$ The pathological changes associated with cerebral small vessel disease include arteriosclerosis, fibrinoid necrosis and lipohyalinosis. $^{6-8}$ These changes lead to cerebral parenchymal damage that is both ischemic and hemorrhagic and based on mechanisms such as chronic hypoperfusion and damage to the blood-brain barrier. ${ }^{9,10}$ The pathogenesis of cerebral small vessel disease is thought to be associated with endothelial dysfunction and inflammation. ${ }^{11,12}$ The severity of this disease is frequently evaluated with neuroimaging techniques such as MRI. Surrogate markers associated with endothelial dysfunction and inflammation that can be used to evaluate the pathophysiology and therapeutic effects of cerebral small vessel disease are necessary.
The ultrasonographic assessment of brachial artery flow-mediated dilation (FMD) is a noninvasive and useful method that has been recently used to measure endothelium-dependent vasodilation. ${ }^{13}$ Several studies have reported that impaired FMD occurs in patients with coronary artery disease and cardiovascular risk factors. ${ }^{14-17}$ Furthermore, a recent systematic review and meta-analysis showed that impaired FMD is a predictor of future cardiovascular events. ${ }^{18}$ However, little is known about the relationship between cerebral small vessel disease and FMD. A study involving 25 older patients with cardiovascular disease reported that the severity of FMD is related to the volume of the WMLs. ${ }^{19}$ However, it is unclear whether FMD is also associated with the presence of CMBs and other biological findings such as inflammation and altered glucose and lipid levels.

The aim of this study was to investigate the association between endothelial function (evaluated by FMD) and the severity of cerebral small vessel disease in patients with chronic cerebrovascular disease and comorbidities.

\footnotetext{
${ }^{1}$ Department of Clinical Neuroscience and Therapeutics, Hiroshima University Graduate School of Biomedical and Health Sciences, Hiroshima, Japan and ${ }^{2}$ Department of Cardiovascular Regeneration and Medicine, Research Center for Radiation Genome Medicine, Research Institute for Radiation Biology and Medicine, Hiroshima University, Hiroshima, Japan

Correspondence: Dr T Nezu, Department of Clinical Neuroscience and Therapeutics, Hiroshima University Graduate School of Biomedical Sciences, 1-2-3 Kasumi, Minami-ku, Hiroshima 734-8551, Japan.

E-mail: tomonezu@hiroshima-u.ac.jp

Received 28 September 2014; revised 9 December 2014; accepted 24 December 2014; published online 12 February 2015
} 


\section{METHODS}

\section{Subjects}

This was a single-center hospital-based prospective study. The study protocol was governed by the guidelines of the national government based on the Helsinki Declaration revised in 1983. The study was approved by the Institutional Research and Ethics Committee of our hospital. All of the patients provided written informed consent to participate in the study. Patients with cerebrovascular disease (at least 6 months after the stroke onset) and other atypical neurological problems who were undergoing a clinically indicated ultrasonographic examination of their carotid artery systems were enrolled between November 2012 and April 2014 at the Hiroshima University Hospital. All of the patients underwent MRI, carotid ultrasonography and FMD studies. Baseline clinical characteristics, including age, sex, hypertension, diabetes mellitus, dyslipidemia, atrial fibrillation, renal dysfunction, history of stroke (ischemic or hemorrhagic), coronary artery disease and current smoking status were recorded. In addition to obtaining a medical history, relevant risk factors were identified from a self-reported medical history or inferred from medications prescribed by the primary physician. Criteria for hypertension, diabetes mellitus and dyslipidemia were previously defined. ${ }^{20}$ Renal functioning was calculated with the estimated glomerular filtration rate (eGFR) using a revised equation for the Japanese population as follows: eGFR $\left(\mathrm{ml} \mathrm{min}^{-1}\right.$ $\left.1.73 \mathrm{~m}^{-2}\right)=194 \times(\text { serum creatinine })^{-1.094} \times(\text { age })^{-0.287} \times 0.739 \quad($ for women). ${ }^{21}$ Renal dysfunction was defined as an eGFR $<60 \mathrm{ml} \mathrm{min}^{-1} 1.73$ $\mathrm{m}^{-2}$. Stroke subtypes were assessed according to the Trial of Org 10172 in Acute Stroke Treatment (TOAST) category. ${ }^{22}$ The carotid ultrasonography, FMD study and blood sample collection were performed in the morning on the same day. The MRI study was performed within 3 months before or after the day when the FMD study was performed.

\section{Carotid ultrasonography}

The common carotid arteries (CCAs) were evaluated using high-resolution B-mode duplex ultrasonography with a $7.5-\mathrm{MHz}$ linear-type probe (Aplio, Toshiba Medicals, Tokyo, Japan). Bilateral optimal visualization of the carotid artery was performed with the patients lying in the supine position with their necks slightly extended. We measured the maximal intima-media thickness (IMT) at the far wall of the distal CCA (CCA-IMT), which is the $10-\mathrm{mm}$ section of the artery proximal to the starting point of the carotid bulb. Plaques in the accessible segments of the CCA and the internal carotid artery were described in terms of plaque scores. ${ }^{23}$ The plaque score is the sum of the heights of all present bilateral plaques. Plaques were defined as IMT $\geqslant 1.1 \mathrm{~mm}$.

\section{FMD and nitroglycerin-mediated vasodilation (NMD)}

The patients were requested to abstain from alcohol, smoking and caffeine on the day of the FMD examination. The FMD examination was conducted during a fasting state in the morning, and only drinking water was given to the patients. Most of the medications taken by the patients were withheld; only those that were deemed necessary (such as antithrombotic therapies) were administered at the discretion of the attending physician. The FMD was measured with a high-resolution linear artery transducer that was coupled to computer-assisted analysis software (UNEXEF38G, UNEX, Nagoya, Japan) as detailed previously. ${ }^{24,25}$ Briefly, the FMD protocol was as follows: a blood pressure cuff was placed around the forearm of the patient. The brachial artery was scanned longitudinally $5-10 \mathrm{~cm}$ above the elbow with a special probe holder (UNEX) to ensure the consistency of the B-mode image. The diameter of the artery was automatically tracked, and the waveform of diameter changes over the cardiac cycle was displayed in real time using the FMD mode of the tracking system. A baseline longitudinal image of the artery was acquired for $30 \mathrm{~s}$ and the blood pressure cuff was inflated to $50 \mathrm{~mm} \mathrm{Hg}$ above systolic pressure for $5 \mathrm{~min}$. Pulsed Doppler velocity signals were obtained for $20 \mathrm{~s}$ at baseline and for $10 \mathrm{~s}$ immediately after cuff deflation. Changes in the diameter of the brachial artery were immediately expressed as the percent change relative to the vessel diameter before cuff inflation. The FMD was automatically calculated as the percent change in peak vessel diameter from the baseline value; \%FMD (peak diameter - baseline diameter/baseline diameter) was used for the analysis. The nitroglycerin response was used to measure endotheliumindependent vasodilation. After acquiring a baseline resting image for $30 \mathrm{~s}$, a $75-\mu \mathrm{g}$ tablet of nitroglycerin was administered sublingually to the patient, and images of the artery were recorded continuously for $8 \mathrm{~min}$. The NMD was automatically calculated as the percent change of the peak vessel diameter from the baseline value; \%NMD (peak diameter-baseline diameter/baseline diameter) was used for the analysis. A single investigator (TN) who was unaware of the clinical details of the patient performed the FMD and NMD evaluations of all of the patients. The intra-observer coefficients of variation were $1.2 \%$ for the baseline brachial artery diameter among all patients and $11.1 \%$ for the FMD in 18 randomly selected patients.

\section{Magnetic resonance imaging}

An MRI was performed with a $1.5 \mathrm{~T}$ scanner (SIGNA, GE Medical Systems, Fairfield, CT, USA or Magneton Symphony Advanced or Avanto, Siemens Medical Systems, Erlangen, Germany) or a $3.0 \mathrm{~T}$ scanner (SIGNA). The imaging protocol consisted of a T1-weighted spin-echo, a T2-weighted spinecho and fluid-attenuated inversion recovery. The severity of WMLs was rated visually from the fluid-attenuated inversion recovery images using the Fazekas scale as follows: no lesions (grade 0), punctate lesions (grade 1), early confluent lesions (grade 2) and confluent lesions (grade 3). ${ }^{26}$ The patients with WMLs of grades 0 to 1 were assigned to the mild WMLs group, and those with WMLs of grades 2 to 3 were assigned to the severe WMLs group. Additionally, a gradientecho T2*-weighted MRI (GRE) was performed to evaluate the presence of CMBs. CMBs were defined as homogeneous round lesions with diameters $\leqslant 10 \mathrm{~mm}$ characterized by signal intensity loss as shown by the GRE. The locations of CMBs were classified as follows: lobar (cortex, subcortex and white matter), deep (basal ganglia, thalamus, brain stem and cerebellum) and mixed (both lobar and deep). Lesions exhibiting signal intensity loss in the globus pallidus or subarachnoid space and diffuse axonal injury were excluded based on appearance or clinical history. ${ }^{27}$ Two stroke neurologists (TN and MA) who were unaware of the clinical details of the patients graded the severity of the WMLs and identified CMBs, respectively.

\section{Statistical analysis}

Statistical analyses were performed using the JMP 10.0 statistical software (SAS Institute, Cary, NC, USA). The data are expressed as the means \pm s.d. or medians (interquartile range) for the continuous variables and as frequencies and percentages for the discrete variables. The statistical significances of the inter-group differences were assessed by $\chi^{2}$ tests, unpaired $t$-tests, the MannWhitney $U$ test or Dunnett's test as appropriate. The relationships between FMD levels and other factors (age, body mass index, CCA-IMT, plaque score, systolic blood pressure, diastolic blood pressure, laboratory findings, brachial artery diameter and NMD) were examined by Spearman's correlation. To obtain the cutoff FMD levels for predicting the severity of WMLs or the presence of $\mathrm{CMBs}$, receiver operating characteristic (ROC) curves were constructed. The areas under the ROC curves were compared with the nonparametric method. ${ }^{28}$ Multiple logistic regression analysis with the backward selection method was performed to determine indicators from age, sex, body mass index, smoking, hypertension, diabetes mellitus, dyslipidemia, atrial fibrillation, renal dysfunction, history of stroke, history of coronary artery disease, CCA-IMT, plaque score, brachial artery diameter and FMD values, that were associated with the severity of WMLs or the presence of CMBs. FMD values were assessed by the cutoff FMD levels based on ROC curves or the 1- $\mathrm{s}$. d. increase variable. In the multivariate model, variables with a $P$-value for the likelihood ratio test $>0.10$ on univariate analysis were excluded. Statistical significance was established at $P<0.05$.

\section{RESULTS}

A total of 102 patients ( 69 males, $70.1 \pm 9.2$ years of age) were enrolled in the study. The baseline clinical characteristics of the patients are presented in Table 1. The univariate regression analysis revealed that FMD was significantly correlated with age $(r=-0.27, P=0.007)$, body mass index $(r=-0.26, P=0.010)$, CCA-IMT $(r=-0.23, P=0.020)$, plaque scores $(r=-0.31, P=0.002)$, eGFR $(r=0.20, P=0.045)$, C-reactive protein levels $(r=-0.25, \quad P=0.013)$, high-density lipoprotein cholesterol levels $(r=0.26, P=0.009)$, triglyceride levels 
Table 1 Baseline characteristics

\begin{tabular}{lc}
\hline & $n=102$ \\
\hline Age (years) & $70.1 \pm 9.2$ \\
Male & $69(67.6)$ \\
BMI (kg m ${ }^{-2}$ ) & $22.8 \pm 3.0$ \\
Smoker & $8(7.8)$ \\
Hypertension & $72(70.6)$ \\
Diabetes mellitus & $30(29.4)$ \\
Dyslipidemia & $63(61.8)$ \\
Atrial fibrillation & $15(14.7)$ \\
Renal dysfunction & $34(33.3)$ \\
SBP (mm Hg) & $132.0 \pm 20.2$ \\
DBP (mm Hg) & $77.8 \pm 12.8$ \\
History of stroke & $72(70.6)$ \\
Cardioembolism & $18(17.6)$ \\
Large-artery atherosclerosis & $10(9.8)$ \\
Small-artery occlusion & $19(18.6)$ \\
Others & $14(13.7)$ \\
Transient ischemic attack & $3(2.9)$ \\
Intracranial hemorrhage & $8(7.8)$ \\
History of coronary artery disease & $10(9.8)$ \\
\end{tabular}

$\begin{array}{ll}\text { MRI findings } & \\ \text { White matter lesions } & 23(22.5) \\ \text { Fazekas Grade 0 } & 39(38.2) \\ \text { Fazekas Grade } 1 & 29(28.4) \\ \text { Fazekas Grade } 2 & 11(10.8) \\ \text { Fazekas Grade } 3 & 30(31.3) \\ \text { Presence of CMBs }(n=96) & 15(15.6) \\ \text { Presence of multiple CMBs }(n=96) & \end{array}$

Physiological findings

CCA-IMT (mm)

Plaque score

Brachial artery diameter ( $\mathrm{mm})$

FMD (\%)

NMD (\%)

$1.71 \pm 0.81$

$4.3(1.5-9.35)$

$4.02 \pm 0.71$

$4.25 \pm 2.15$

$13.30 \pm 5.04$

$\begin{array}{lc}\text { Laboratory findings } & \\ \text { CRP, log }\left(\mathrm{mg} \mathrm{dl}^{-1}\right) & -1.18 \pm 0.55 \\ \text { HbAlc }(\%) & 5.95 \pm 0.75 \\ \left.\text { FBG }(\mathrm{mg} \mathrm{dl})^{-1}\right) & 111.8 \pm 24.3 \\ \text { LDL cholesterol }\left(\mathrm{mg} \mathrm{dl}^{-1}\right) & 109.4 \pm 26.3 \\ \text { HDL cholesterol }\left(\mathrm{mg} \mathrm{dl}^{-1}\right) & 63.9 \pm 21.3 \\ \text { TG }\left(\mathrm{mg} \mathrm{dl}^{-1}\right) & 105.6 \pm 46.1 \\ \text { eGFR }\left(\mathrm{ml} \mathrm{min}^{-1} 1.73 \mathrm{~m}^{-2}\right) & 66.9 \pm 17.5\end{array}$

Medications

Antiplatelet agent

55 (53.9)

Anticoagulant agent

24 (23.5)

ACE inhibitor or ARB

$43(42.2)$

35 (34.3)

Calcium channel blocker

16 (15.7)

Statin

52 (51.0)

26 (25.5)

Abbreviations: ACE, angiotensin-converting enzyme; ARB, angiotensin II receptor blocker; BMI, body mass index; CCA, common carotid arteries; CMBs, cerebral microbleeds; CRP, C-reactive protein; DBP, diastolic blood pressure; eGFR, estimated glomerular filtration rate; FBG, fasting blood glucose; FMD, flow-mediated dilation; HDL, high-density lipoprotein; IMT, intima-media thickness; LDL, low-density lipoprotein; NMD, nitroglycerin-mediated dilation; SBP, systolic blood pressure; TG, triglycerides.

The data are presented as the mean \pm s.d. for age, BMI, SBP, DBP, CCA-IMT, brachial artery diameter, FMD, NMD and laboratory findings, medians (interquartile ranges) for plaque score, and numbers of patients (\%) for the others. $(r=-0.28, P=0.004)$, brachial artery diameter $(r=-0.34, P<0.001)$ and NMD $(r=0.40, P<0.001)$.

Of 102 patients, 61 patients had a history of ischemic stroke, 3 patients had a history of transient ischemic attack, and 8 patients had a history of intracranial hemorrhage caused by hypertension. There was no significant difference in FMD values among the patients according to stroke subtypes (non-stroke, $4.61 \pm 2.31 \%$; cardioembolism, $\quad 4.93 \pm 2.47 \%$; large-artery atherosclerosis, $3.80 \pm 1.94 \%$; small-artery occlusion, $3.55 \pm 1.40 \%$; others, $4.71 \pm 2.26 \%$; transient ischemic attack, $3.00 \pm 0.92 \%$; intracranial hemorrhage, $3.28 \pm 2.14 \%$; $P=0.210$, respectively). On the other hand, the patients with smallartery occlusion or intracranial hemorrhage $(n=27)$ had significantly impaired FMD compared with the other patients $(3.47 \pm 1.61$ vs. $4.53 \pm 2.26 \%, P=0.027)$. Although there was no significant difference in WMLs between the patients with small-artery occlusion or intracranial hemorrhage and those without (Fazekas grading scores, median [interquartile range] 2 [1-2] vs. 1 [0-2], $P=0.099)$, the patients with small-artery occlusion or intracranial hemorrhage had higher rates of the presence of CMBs compared with the other patients (54.2 vs. $23.6 \%, P=0.010$ ).

\section{Associations between FMD and WMLs}

FMD was associated with the severity of WMLs (Figure 1). The patients were assigned to the severe WMLs group or the mild WMLs group (Table 2). The patients in the severe WMLs group were older $(P=0.001)$ and more frequently exhibited hypertension $(P=0.045)$ and diabetes mellitus $(P=0.026)$ than the patients in the mild WMLs group. Serum levels of $\mathrm{HbAlc}$ and fasting blood glucose were higher in the patients with severe WMLs than in the patients with mild WMLs $(P=0.012$ and $P=0.016$, respectively). A greater impairment in FMD was found in the patients with severe WMLs than in the patients with mild WMLs $(P<0.001)$; however, there was no significant difference in NMD between the two groups. There were no significant differences in medication use between the groups. The optimal FMD cutoff value for predicting the severity of WMLs was 3.9, with a sensitivity of $82.5 \%$ and a specificity of $66.1 \%$. The area under the ROC curve was significantly larger for FMD $(0.775,95 \%$ confidence interval (CI) $0.670-0.854)$ than for $\mathrm{NMD}(0.598,95 \%$ CI $0.484-0.703$ ) (Figure 2a, $P=0.004$ ). The multivariate logistic analysis indicated that increased age (odds ratio (OR) $1.11,95 \% \mathrm{CI}$ 1.04-1.19) and FMD <4.0\% (OR 9.50, 95\% CI 3.55-28.83) were independently associated with the severity of WMLs. FMD values (per 1 -s.d. increase) were also associated with the severity of WMLs after multiple logistic regression analysis (OR 0.34, 95\% CI 0.17-0.63).

\section{Associations between FMD and CMBs}

A gradient-echo $\mathrm{T} 2{ }^{*}$-weighted MRI (GRE) was performed in 96 patients (94.1\%). CMBs were observed in 30 of these patients $(31.3 \%)$; 15 patients had a single $\mathrm{CMB}$ and 15 had multiple CMBs. These CMBs were found in deep locations, lobar locations and mixed locations ( $n=14,9$ and 7, respectively). FMD values for the patients are shown in Figure 3. The 96 patients were classified as either having or lacking CMBs (Table 2). The patients with CMBs were older $(P=0.036)$ and more frequently afflicted with hypertension $(P=0.016)$ than those without CMBs. The systolic blood pressure, plaque score and brachial artery diameter were higher in the patients with $\mathrm{CMBs}$ than in the patients without $\mathrm{CMBs}(P=0.016, P=0.020$ and $P=0.021$, respectively). Additionally, FMD and NMD were lower in the patients with CMBs than in the patients without CMBs $(P=0.002$ and $P<0.001$, respectively). There were no significant differences in medication use, including antithrombotic agents, between the groups. The optimal 
FMD cutoff value for predicting the presence of CMBs was 3.7, with a sensitivity of $73.3 \%$ and a specificity of $68.2 \%$. The area under the ROC curve for FMD $(0.719,95 \%$ CI $0.599-0.815)$ was similar to that

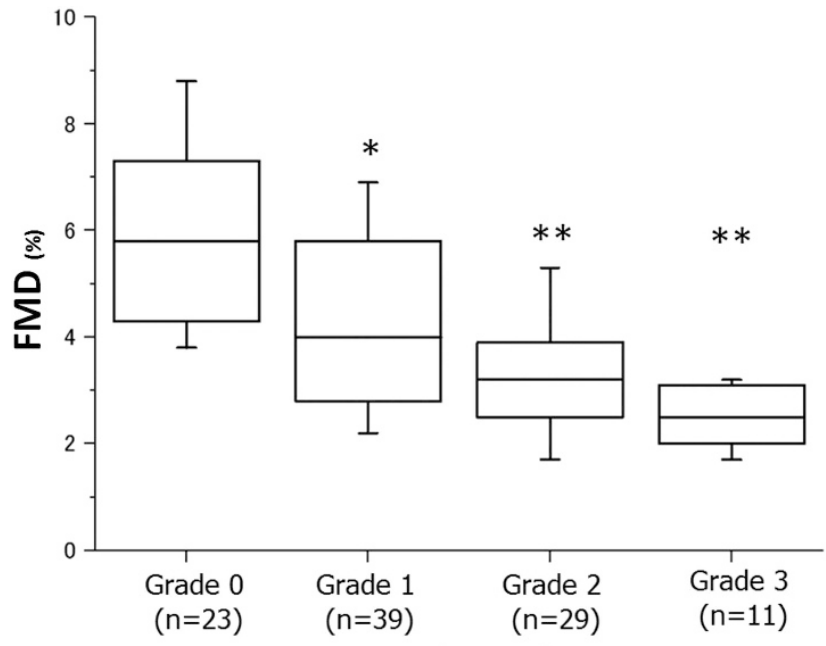

Fazekas scale

Figure 1 Association between endothelial function as measured by flowmediated dilation (FMD) and the severity of white matter lesions according to the Fazekas scale (grades $0,1,2$ and 3 ). The bottom and top of the box represent the 25th and 75th percentiles, respectively; the upper and lower lines indicate the 10th and 90th percentiles, respectively. The band near the middle of the box marks the median value. ${ }^{*} P<0.050$ vs. grade 0 as determined by Dunnett's test. ${ }^{* *} P<0.001$ vs. grade 0 as determined by Dunnett's test. obtained for NMD (0.755, 95\% CI 0.639-0.843) (Figure 2b, $P=0.557)$. The multivariate logistic analysis showed that the presence of hypertension (OR 4.14, 95\% CI 1.31-16.12, $P=0.024$ ) and FMD $<3.8 \%$ (OR 5.82, 95\% CI 2.23-16.50, $P=0.001$ ) were independently associated with the presence of CMBs. FMD values (per 1-s.d. increase) were slightly associated, although not significantly, with the presence of $\mathrm{CMBs}$ after multiple logistic regression analysis (OR 0.10 , 95\% CI $0.01-1.17, P=0.068$ ).

\section{DISCUSSION}

We found that impaired FMD was independently associated with the severity of WMLs and the presence of CMBs in patients with chronic cerebrovascular disease and comorbidities. Our results suggest that endothelial dysfunction measured by FMD might be associated with the pathogenesis of cerebral small vessel disease, independent of classical cardiovascular risk factors.

Studies using positron emission tomography have suggested that WMLs are caused by chronic hypoperfusion associated with the progression of cerebral small-artery damage. ${ }^{29,30}$ In addition, the impairment of vascular reactivity is thought to contribute to the development of WMLs. Although studies evaluating cerebral vascular reactivity have been performed to elucidate the association between cerebral vascular reactivity and WMLs, this association remains controversial. ${ }^{31-36}$ These inconsistencies may have arisen because different modalities have been used to evaluate cerebral vascular reactivity, including transcranial Doppler ultrasound, perfusion MRI, xenon inhalation computed tomography, single photon emission computed tomography, positron emission tomography and vasodilatory stimulus methods such as $\mathrm{CO}_{2}$ inhalation, breath-holding hyperventilation tests and the acetazolamide challenge test. ${ }^{31}$

Table 2 Baseline characteristics of patients assigned to different groups based on the severity of their WMLs and the presence of CMBs

\begin{tabular}{|c|c|c|c|c|}
\hline & \multicolumn{2}{|c|}{$W M L s$} & \multicolumn{2}{|c|}{$C M B s$} \\
\hline & Mild WMLs $\mathrm{n}=62$ & Severe $W M L s \mathrm{n}=40$ & $C M B s(-) n=66$ & $C M B s(+) \mathrm{n}=30$ \\
\hline Age (years) & $67.6 \pm 9.9^{\ddagger}$ & $74.0 \pm 6.4$ & $68.6 \pm 9.7^{*}$ & $72.8 \pm 7.4$ \\
\hline Male & $40(64.5)$ & $29(73.0)$ & $40(60.6)$ & $24(80.0)$ \\
\hline $\mathrm{BMI}\left(\mathrm{kg} \mathrm{m}^{-2}\right)$ & $22.2 \pm 3.9$ & $23.2 \pm 3.1$ & $22.5 \pm 2.7$ & $23.2 \pm 3.6$ \\
\hline Smoker & $5(8.1)$ & $3(7.5)$ & $7(10.6)$ & $1(3.3)$ \\
\hline Hypertension & $39(62.9)^{*}$ & $33(82.5)$ & $40(60.6)^{*}$ & $26(86.7)$ \\
\hline Diabetes mellitus & $13(21.0)^{*}$ & $17(42.5)$ & $17(25.8)$ & $10(33.3)$ \\
\hline Dyslipidemia & $39(62.9)$ & $24(60.0)$ & $41(62.1)$ & $18(60.0)$ \\
\hline Atrial fibrillation & $10(16.1)$ & $5(12.5)$ & $10(15.2)$ & $4(13.3)$ \\
\hline Renal dysfunction & $18(29.0)$ & $16(40.0)$ & $23(34.9)$ & $9(30.0)$ \\
\hline SBP $(\mathrm{mm} \mathrm{Hg})$ & $129.1 \pm 20.9$ & $136.6 \pm 18.4$ & $129.0 \pm 21.1^{*}$ & $139.8 \pm 17.7$ \\
\hline DBP (mm Hg) & $77.1 \pm 13.5$ & $78.9 \pm 11.7$ & $77.4 \pm 12.4$ & $79.7 \pm 14.3$ \\
\hline History of stroke & $44(7 \overline{1} .0)$ & $28(70.0)$ & $43(6 \overline{5} .2)$ & $24(80.0)$ \\
\hline History of coronary artery disease & $4(6.5)$ & $6(15.0)$ & $5(7.6)$ & $5(16.7)$ \\
\hline \multicolumn{5}{|l|}{ Physiological findings } \\
\hline CCA-IMT (mm) & $1.68 \pm 0.85$ & $1.76 \pm 0.76$ & $1.68 \pm 0.85$ & $1.86 \pm 0.76$ \\
\hline Plaque score & $3.3(1.25-8.75)$ & $6.75(2.53-10.75)$ & $3.3(1.4-7.45)^{*}$ & $7.65(3.3-13.53)$ \\
\hline Brachial artery diameter (mm) & $3.94 \pm 0.62$ & $4.15 \pm 0.81$ & $3.89 \pm 0.67 *$ & $4.25 \pm 0.72$ \\
\hline FMD (\%) & $4.99 \pm 2.28^{\S}$ & $3.12 \pm 1.30$ & $4.81 \pm 2.27^{\dagger}$ & $3.36 \pm 1.47$ \\
\hline NMD (\%) & $14.09 \pm 5.51$ & $12.12 \pm 4.03$ & $14.86 \pm 4.88^{\S}$ & $10.26 \pm 4.04$ \\
\hline \multicolumn{5}{|l|}{ Laboratory findings } \\
\hline CRP, log (mg dl-1) & $-1.24 \pm 0.52$ & $-1.08 \pm 0.60$ & $-1.20 \pm 0.55$ & $-1.09 \pm 0.59$ \\
\hline $\mathrm{HbAlc}(\%)$ & $5.81 \pm 0.68 *$ & $6.19 \pm 0.81$ & $5.89 \pm 0.66$ & $6.08 \pm 0.95$ \\
\hline FBG $\left(\mathrm{mg} \mathrm{dl}^{-1}\right)$ & $107.1 \pm 20.4^{*}$ & $119.0 \pm 28.1$ & $105.9 \pm 26.0$ & $109.3 \pm 32.4$ \\
\hline LDL cholesterol (mg dl-1) & $111.4 \pm 28.2$ & $106.3 \pm 23.0$ & $110.7 \pm 26.3$ & $111.0 \pm 26.7$ \\
\hline HDL cholesterol (mg dl-1) & $66.7 \pm 22.9$ & $59.5 \pm 17.9$ & $64.2 \pm 23.7$ & $64.8 \pm 15.3$ \\
\hline $\mathrm{TG}\left(\mathrm{mg} \mathrm{dl}^{-1}\right)$ & $102.3 \pm 42.3$ & $110.7 \pm 51.6$ & $108.2 \pm 46.7$ & $102.2 \pm 37.5$ \\
\hline eGFR (ml min $\left.{ }^{-1} 1.73 \mathrm{~m}^{-2}\right)$ & $66.9 \pm 17.1$ & $67.0 \pm 19.2$ & $67.5 \pm 15.4$ & $65.9 \pm 22.4$ \\
\hline
\end{tabular}

Abbreviations: BMI, body mass index; CMBs, cerebral microbleeds; CRP, C-reactive protein; DBP, diastolic blood pressure; eGFR, estimated glomerular filtration rate; FBG, fasting blood glucose; FMD, flow-mediated dilation; HDL, high-density lipoprotein; IMT, intima media thickness; LDL, low-density lipoprotein; NMD, nitroglycerin-mediated dilation; SBP, systolic blood pressure; TG,

triglycerides; WML, white matter lesions. 

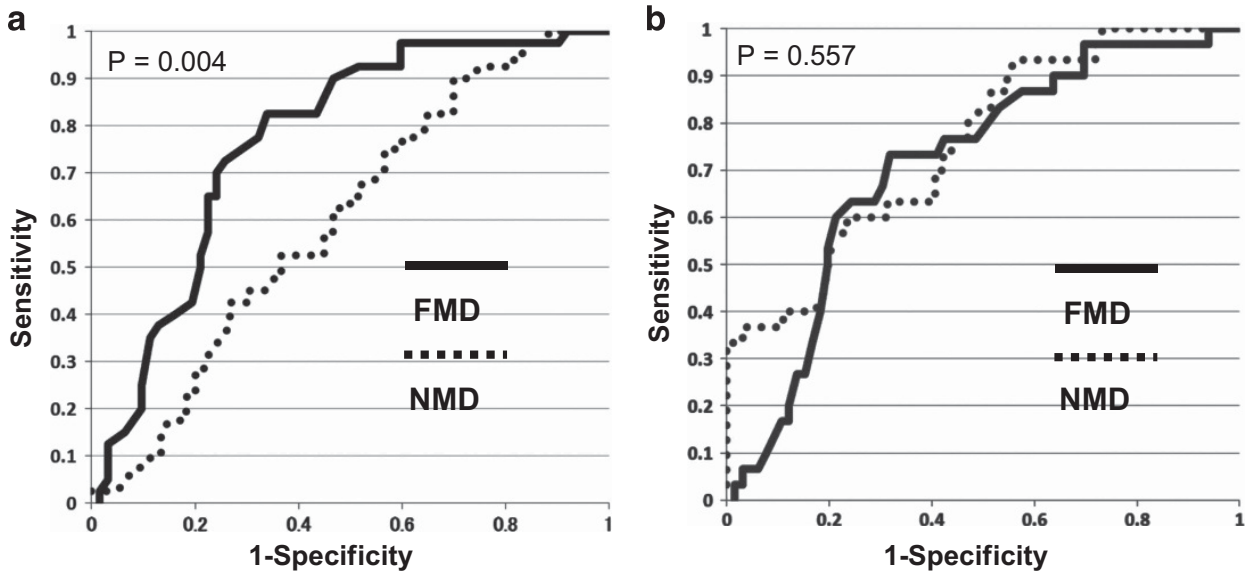

Figure 2 (a) Receiver operating characteristic (ROC) curves of flow-mediated dilation (FMD) or nitroglycerin-mediated dilation (NMD) for predicting the severity of white matter lesions. (b) ROC curves of FMD or NMD for predicting the presence of cerebral microbleeds.

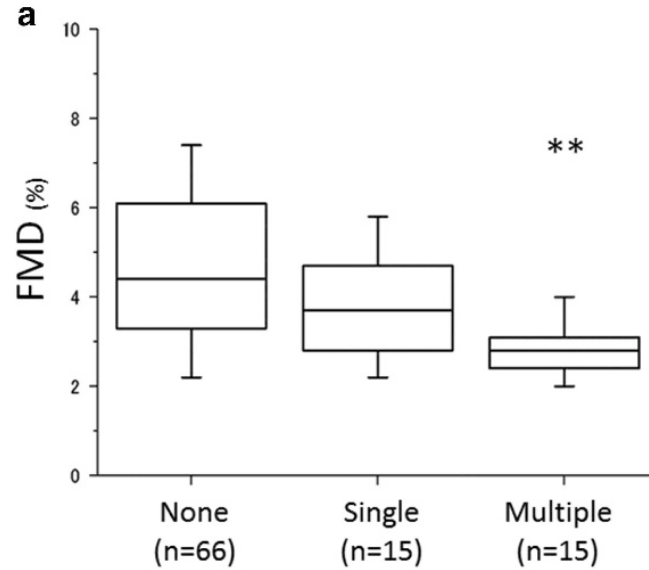

Cerebral microbleeds b

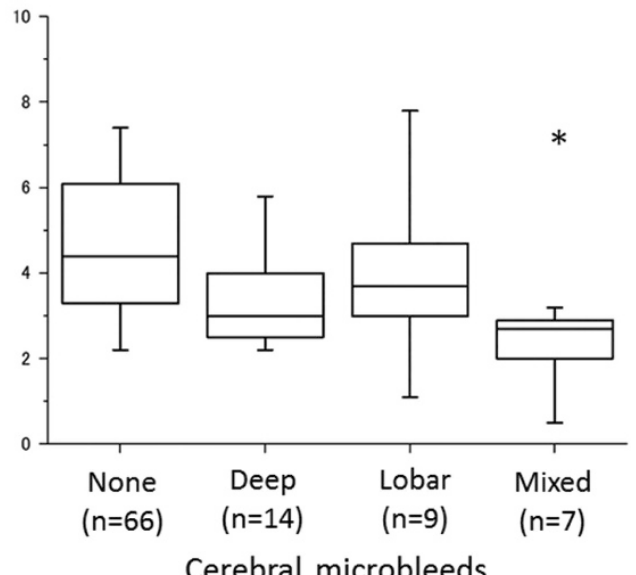

Cerebral microbleeds

Figure 3 (a) Association between endothelial function as measured by flow-mediated dilation (FMD) and the number of cerebral microbleeds. (b) Association between endothelial functioning as measured by FMD and the locations of cerebral microbleeds. The bottom and top of the box represent the 25th and 75th percentiles, respectively; the upper and lower lines indicate the 10th and 90th percentiles, respectively. The band near the middle of the box marks the median value. ${ }^{*} P<0.050$ vs. none as determined by Dunnett's test. ${ }^{*} P<0.010$ vs. none as determined by Dunnett's test.

Moreover, the presence of major cerebral artery occlusive disease, which has been strongly associated with impaired cerebral vascular reactivity, should be considered. ${ }^{37}$ FMD has recently been used to measure endothelium-dependent vasodilation in the brachial artery to predict not only cardiovascular events but also systematic vascular damage. ${ }^{13,18}$ The pathogenesis of cerebral small vessel disease, including the occurrence of WMLs, is also thought to be associated with endothelial dysfunction. Peripheral vascular reactivity measured by FMD differs from $\mathrm{CO}_{2}$ reactivity or acetazolamide reactivity because FMD reflects the intrinsic nitric oxide bioavailability of the vascular endothelium. Only one study involving 25 older patients with cardiovascular disease has described an association between impaired FMD and the severity of WMLs. ${ }^{19}$ Our studies have provided additional evidence of an association between endothelial dysfunction and the development of WMLs. Further studies assessing the association between FMD and cerebral vascular reactivity and the suitability of FMD or cerebral vascular reactivity for the evaluation of the pathophysiology of WMLs are needed.
Lacunar infarction, such as a small-artery occlusion, is one of the cerebral small vessel diseases. Chen et al. ${ }^{38}$ observed that acute ischemic stroke patients with lacunar infarction had significantly impaired FMD compared with controls. Hypertensive intracranial hemorrhage is also a cerebral small vessel disease. The present study showed that the patients with small-artery occlusion or intracranial hemorrhage had significantly impaired FMD compared with the other patients. Another study reported that 18 patients with a chronic lacunar infarction ( $>4$ weeks after stroke onset) experienced impaired FMD. ${ }^{39}$ The authors of this study also evaluated cerebral vascular reactivity to L-arginine as measured by transcranial Doppler ultrasound (which is thought to reflect cerebral endothelial functioning) and found that L-arginine reactivity is also decreased in patients with a lacunar infarction. Interestingly, both L-arginine reactivity and FMD improved after 3 months of atorvastatin treatment. FMD may be a surrogate marker that represents a therapeutic target for cerebral small vessel disease, even though the severity of WMLs was not assessed in these previous studies. 
In the present study, impaired FMD was independently associated not only with the severity of WMLs but also with the presence of CMBs. The analysis of ROC curves showed that NMD was useful for predicting the presence of CMBs, as well as FMD. Because both endogenous nitric oxide and administered nitroglycerin act on vascular smooth muscle cells, NMD, which is an index of endothelium-independent vasodilation and is assessed following the sublingual administration of nitroglycerin, has been used as a positive control measure when evaluating FMD to differentiate between endothelium-dependent and endothelium-independent vasodilation. ${ }^{40}$ Maruhashi et al. ${ }^{40}$ showed that FMD should be interpreted as an index of vascular function reflecting both endothelium-dependent vasodilation and endothelium-independent vasodilation in subjects with impaired NMD. Pathological changes associated with cerebral small vessel disease can lead to ischemic consequences such as WMLs or lacunar infarctions and hemorrhagic lesions such as CMBs. ${ }^{2}$ Hemorrhagic changes such as CMBs that occur in patients with small vessel disease might be associated with damage to vascular smooth muscle cells caused by endothelial dysfunction, inflammation and the disruption of the blood-brain barrier. Indeed, vasculopathy is characterized by the degeneration of vascular smooth muscle cells and the accumulation of granular osmiophilic material at the surfaces of these cells in patients with cerebral autosomal dominant arteriopathy with subcortical infarcts. ${ }^{41}$ Moreover, amyloid $\beta$ deposits were found in the vessel walls and associated with the vulnerabilities of the cerebral small vessels in patients with cerebral amyloid angiopathy. ${ }^{42}$ The present study suggests that the pathophysiology underlying CMBs is not only associated with endothelial dysfunction but also with vascular smooth muscle cell dysfunction.

This study has several limitations. First, our study had a small sample size and a cross-sectional design, which limited our ability to make causal inferences. In addition, because only patients who required an ultrasonographic examination of their carotid artery systems were recruited in this study, most of the patients had a history of stroke and had taken antiplatelet or anticoagulant medication. Therefore, the prevalence of CMBs was higher in the patients in this study $(31.3 \%)$ than in the general population, ${ }^{2}$ which may have caused selection bias. Second, we could not identify CMBs in all of the patients using the same MRI modality. MRI characteristics such as pulse sequence, sequence parameters and magnetic field strength may have affected the subtle appearances of the CMBs. Third, certain medications (such as antithrombotic therapies) that, if withheld, could have resulted in cardiovascular events were administered to the patients. However, patients who received nitrates were not included, which might have mitigated the impact of this issue. Although various medications can modify endothelial functioning, our findings demonstrated that impaired FMD was associated with severe cerebral small vessel disease in real clinical situations.

In conclusion, endothelial dysfunction as measured by FMD was associated with the severity of cerebral small vessel disease in patients with chronic cerebrovascular disease and vascular risk factors. FMD shows promise as a surrogate marker for the evaluation of the pathophysiology of cerebral small vessel disease. A confirmation of the present findings using patients in the general population is necessary.

\section{ACKNOWLEDGEMENTS}

We express our gratitude to Takamichi Sugimoto, Shigeru Tanaka, Tomokazu Nishikawa, Hiroki Fujii, Hayato Matsushima, Yasuko Furuno, Miwako Sasanishi and Akemi Hironaka for their clinical research advice, ultrasonographic and technical assistance. This study was supported in part by research grants from the Smoking Research Foundation, the Tsuchiya Foundation, the Japan Science and Technology Agency and the Japan Heart Foundation.

1 Pantoni L, Garcia JH. Pathogenesis of leukoaraiosis: A review. Stroke 1997; 28 652-659.

2 Pantoni L. Cerebral small vessel disease: From pathogenesis and clinical characteristics to therapeutic challenges. Lancet Neurol 2010; 9: 689-701.

3 Kalaria RN. Cerebrovascular disease and mechanisms of cognitive impairment Evidence from clinicopathological studies in humans. Stroke 2012; 43: 2526-2534.

4 Palumbo V, Boulanger JM, Hill MD, Inzitari D, Buchan AM, CASES Investigators. Leukoaraiosis and intracerebral hemorrhage after thrombolysis in acute stroke. Neurology 2007; 68: 1020-1024.

5 Neumann-Haefelin T, Hoelig S, Berkefeld J, Fiehler J, Gass A, Humpich M, Kastrup A, Kucinski T, Lacei O, Liebeskind DS, Rother J, Rosso C, Samson Y, Saver JL, Yan B, MR Stroke Group. Leukoaraiosis is a risk factor for symptomatic intracerebral hemorrhage after thrombolysis for acute stroke. Stroke 2006; 37: 2463-2466.

6 Lammie GA. Hypertensive cerebral small vessel disease and stroke. Brain Pathol 2002; 12: 358-370.

7 Grinberg LT, Thal DR. Vascular pathology in the aged human brain. Acta Neuropathol 2010; 119: 277-290.

8 Isa K, Sakima A, Sakima H, Nakachi K, Kinjyo K, Ohya Y. Association between the intima-media thickness of the brachiocephalic trunk and white matter hyperintensity in brain MRI. Hypertens Res 2013; 36: 980-984.

9 Wardlaw JM, Sandercock PA, Dennis MS, Starr J. Is breakdown of the blood-brain barrier responsible for lacunar stroke, leukoaraiosis, and dementia? Stroke 2003; 34: 806-812.

10 Yao H, Sadoshima S, Ibayashi S, Kuwabara Y, Ichiya Y, Fujishima M. Leukoaraiosis and dementia in hypertensive patients. Stroke 1992; 23: 1673-1677.

11 Fornage M, Chiang YA, O'Meara ES, Psaty BM, Reiner AP, Siscovick DS, Tracy RP, Longstreth WT Jr. Biomarkers of inflammation and MRI-defined small vessel disease of the brain: The Cardiovascular Health Study. Stroke 2008; 39: 1952-1959.

12 Stevenson SF, Doubal FN, Shuler K, Wardlaw JM. A systematic review of dynamic cerebral and peripheral endothelial function in lacunar stroke versus controls. Stroke 2010; 41: e434-e442.

13 Celermajer DS, Sorensen KE, Gooch VM, Spiegelhalter DJ, Miller OI, Sullivan ID, Lloyd JK, Deanfield JE. Non-invasive detection of endothelial dysfunction in children and adults at risk of atherosclerosis. Lancet 1992; 340: 1111-1115.

14 Suwaidi JA, Hamasaki S, Higano ST, Nishimura RA, Holmes DR, Lerman A. Long-term follow-up of patients with mild coronary artery disease and endothelial dysfunction. Circulation 2000; 101: 948-954.

15 Schächinger V, Britten MB, Zeiher AM. Prognostic impact of coronary vasodilator dysfunction on adverse long-term outcome of coronary heart disease. Circulation 2000; 101: 1899-1906.

16 Gokce N, Keaney JF, Hunter LM, Watkins MT, Menzoian JO, Vita JA. Risk stratification for postoperative cardiovascular events via noninvasive assessment of endothelial function: A prospective study. Circulation 2002; 105: 1567-1572.

17 Östlund E, Al-Nashi M, Hamad RR, Larsson A, Eriksson M, Bremme K, Kahan T. Normalized endothelial function but sustained cardiovascular risk profile 11 years following a pregnancy complicated by preeclampsia. Hypertens Res 2013; 36: 1081-1087.

18 Ras RT, Streppel MT, Draijer R, Zock PL. Flow-mediated dilation and cardiovascular risk prediction: A systematic review with meta-analysis. Int J Cardiol 2013; 168: 344-351.

19 Hoth KF, Tate DF, Poppas A, Forman DE, Gunstad J, Moser DJ, Paul RH, Jefferson AL, Haley AP, Cohen RA. Endothelial function and white matter hyperintensities in older adults with cardiovascular disease. Stroke 2007; 38: 308-312.

20 Hosomi N, Aoki S, Matsuo K, Deguchi K, Masugata H, Murao K, Ichihara N, Ohyama H, Dobashi H, Nezu T, Ohtsuki T, Yasuda O, Soejima H, Ogawa H, Izumi Y, Kohno M, Tanaka J, Matsumoto M. Association of serum anti-periodontal pathogen antibody with ischemic stroke. Cerebrovasc Dis 2012; 34: 385-392.

21 Matsuo S, Imai E, Horio M, Yasuda Y, Tomita K, Nitta K, Yamagata K, Tomino Y, Yokoyama $\mathrm{H}$, Hishida A. Collaborators developing the Japanese equation for estimated GFR. Revised equations for estimated GFR from serum creatinine in Japan. Am J Kidney Dis 2009; 53: 982-992.

22 Adams HP, Bendixen BH, Kappelle LJ, Biller J, Love BB, Gordon DL, March EE 3rd. Classification of subtype of acute ischemic stroke. Definitions for use in a multicente clinical trial. Toast. Trial of org 10172 in acute stroke treatment. Stroke 1993; 24 35-41.

23 Handa N, Matsumoto M, Maeda H, Hougaku H, Kamada T. Ischemic stroke events and carotid atherosclerosis. Results of the Osaka Follow-up Study for Ultrasonographic Assessment of Carotid Atherosclerosis (the OSACA Study). Stroke 1995; 26: $1781-1786$

24 Maruhashi T, Soga J, Fujimura N, Idei N, Mikami S, Iwamoto Y, Kajikawa M, Matsumoto T, Kihara Y, Chayama K, Noma K, Nakashima A, Tomiyama H Takase B, Yamashina A, Higashi Y. Hyperbilirubinemia, augmentation of endothelial function, and decrease in oxidative stress in Gilbert syndrome. Circulation 2012; 126: 598-603. 
25 Iwamoto Y, Maruhashi T, Fujii Y, Idei N, Fujimura N, Mikami S, Kajikawa M, Matsumoto T, Kihara Y, Chayama K, Noma K, Nakashima A, Higashi Y. Intima-media thickness of brachial artery, vascular function, and cardiovascular risk factors. Arterioscler Thromb Vasc Biol 2012; 32: 2295-2303.

26 Fazekas F, Chawluk JB, Alavi A, Hurtig HI, Zimmerman RA. MR signal abnormalities at $1.5 \mathrm{~T}$ in Alzheimer's dementia and normal aging. Am J Roentgenol 1987; 149: 351-356.

27 Greenberg SM, Vernooij MW, Cordonnier C, Viswanathan A, AI-Shahi Salman R, Warach S, Launer LJ, Van Buchem MA, Breteler MM, Microbleed Study Group. Cerebral microbleeds: A guide to detection and interpretation. Lancet Neurol 2009; 8 : 165-174.

28 DeLong ER, DeLong DM, Clarke-Pearson DL. Comparing the areas under two or more correlated receiver operating characteristic curves: A nonparametric approach. Biometrics 1988; 44: 837-845.

29 Meguro K, Hatazawa J, Yamaguchi T, Itoh M, Matsuzawa T, Ono S, Miyazawa H, Hishinuma T, Yanai K, Sekita Y, Yamada K. Cerebral circulation and oxygen metabolism associated with subclinical periventricular hyperintensity as shown by magnetic resonance imaging. Ann Neurol 1990; 28: 378-383.

30 Yao H, Sadoshima S, Kuwabara Y, Ichiya Y, Fujishima M. Cerebral blood flow and oxygen metabolism in patients with vascular dementia of the Binswanger type. Stroke 1990; 21: 1694-1699.

31 Nezu T, Yokota C, Uehara T, Yamauchi M, Fukushima K, Toyoda K, Matsumoto M, lida $\mathrm{H}$, Minematsu K. Preserved acetazolamide reactivity in lacunar patients with severe white-matter lesions: ${ }^{15} \mathrm{O}$-labeled gas and $\mathrm{H}_{2} \mathrm{O}$ positron emission tomography studies. J Cereb Blood Flow Metab 2012; 32: 844-850.

32 Birns J, Jarosz J, Markus HS, Kalra L. Cerebrovascular reactivity and dynamic autoregulation in ischaemic subcortical white matter disease. J Neurol Neurosurg Psychiatry 2009; 80: 1093-1098.

33 Bakker SL, de Leeuw FE, de Groot JC, Hofman A, Koudstaal PJ, Breteler MM. Cerebral vasomotor reactivity and cerebral white matter lesions in the elderly. Neurology 1999; 52: 578-583.

34 Fu JH, Lu CZ, Hong Z, Dong Q, Ding D, Wong KS. Relationship between cerebral vasomotor reactivity and white matter lesions in elderly subjects without large artery occlusive disease. J Neuroimaging 2006; 16: 120-125.
35 Kozera GM, Dubaniewicz M, Zdrojewski T, Madej-Dmochowska A, Mielczarek M, Wojczal J, Chwojnicki K, Swierblewska E, Schminke U, Wyrzkowski B, Nyka WM, SOPKARD Study Group. Cerebral vasomotor reactivity and extent of white matter lesions in middle-aged men with arterial hypertension: A pilot study. Am J Hypertens 2010; 23: 1198-1203.

36 Maeda H, Matsumoto M, Handa N, Hougaku H, Ogawa S, Itoh T, Tsukamoto Y, Kamada T. Reactivity of cerebral blood flow to carbon dioxide in various types of ischemic cerebrovascular disease: Evaluation by the transcranial Doppler method. Stroke 1993; 24: 670-675.

37 Hirano T, Minematsu K, Hasegawa $Y$, Tanaka $Y$, Hayashida K, Yamaguchi T. Acetazolamide reactivity on $123^{l}-$ IMP single photon emission computed tomography in patients with major cerebral artery occlusive disease: Correlation with positron emission tomography parameters. J Cereb Blood Flow Metab 1994; 14: 763-770.

38 Chen PL, Wang PY, Sheu WH, Chen YT, Ho YP, Hu HH, Hsu HY. Changes of brachial flow-mediated vasodilation in different ischemic stroke subtypes. Neurology 2006; 67: 1056-1058.

39 Pretnar-Oblak J, Sabovic M, Sebestjen M, Pogacnik T, Zaletel M. Influence of atorvastatin treatment on $\mathrm{L}$-arginine cerebrovascular reactivity and flow-mediated dilatation in patients with lacunar infarctions. Stroke 2006; 37: 2540-2545.

40 Maruhashi T, Soga J, Fujimura N, Idei N, Mikami S, Iwamoto Y, Kajikawa M, Matsumoto T, Hidaka T, Kihara Y, Chayama K, Noma K, Nakashima A, Goto C, Higashi $Y$. Nitroglycerine-induced vasodilation for assessment of vascular function: a comparison with flow-mediated vasodilation. Arterioscler Thromb Vasc Biol 2013; 33: 1401-1408.

41 Joutel A, Andreux F, Gaulis S, Domenga V, Cecillon M, Battail N, Piga N, Chapon F, Godfrain C, Tournier-Lasserve E. The ectodomain of the Notch3 receptor accumulates within the cerebrovasculature of CADASIL patients. J Clin Invest 2000; 105: 597-605.

42 Schrag M, McAuley G, Pomakian J, Jiffry A, Tung S, Mueller C, Vinters HV, Haacke EM, Holshouser B, Kido D, Kirsh WM. Correlation of hypointensities in susceptibility-weighted images to tissue histology in dementia patients with cerebral amyloid angiopathy: A postmortem MRI study. Acta Neuropathol 2010; 119: 291-302. 